\title{
Heterogeneity of Glycan Processing on Trimeric SARS-CoV-2 Spike Protein Revealed by Charge Detection Mass Spectrometry
}

Lohra M. Miller, ${ }^{a}$ Lauren F. Barnes, ${ }^{a}$ Shannon A. Raab, ${ }^{a}$ Benjamin E. Draper, ${ }^{b}$ Tarick J. ElBaba, ${ }^{\mathrm{c}}$ Corinne A. Lutomski, ${ }^{\mathrm{c}}$ Carol V. Robinson, ${ }^{\mathrm{c}}$ David E. Clemmer, ${ }^{\mathrm{a}}$ and Martin F. Jarrold ${ }^{\mathrm{a}}$

${ }^{a}$ Chemistry Department, Indiana University, 800 E Kirkwood Ave, Bloomington Indiana 47405

${ }^{b}$ Megadalton Solutions, 3520 E Bluebird Ln, Bloomington Indiana 47401

${ }^{c}$ Department of Chemistry, University of Oxford, South Parks Road, Oxford, OXI 3QZ, UK

Supporting Information 


\section{CDMS Mass Resolution with 100 ms Trapping Time}

In CDMS, the mass resolution has contributions from the $\mathrm{m} / \mathrm{z}$ resolution and the charge resolution:

$$
\frac{\Delta m}{m}=\sqrt{\left(\frac{\Delta[m / z]}{[m / z]}\right)^{2}+\left(\frac{\Delta z}{z}\right)^{2}}
$$

Where $\Delta m, \Delta[m / z]$, and $\Delta z$ are the peak full widths at half maximum (FWHM) in the mass, $m / z$, and charge distributions. The $100 \mathrm{~ms}$ trapping measurements reported in the manuscript were performed on our prototype CDMS instrument. ${ }^{1}$ On this instrument, the relative root mean square deviation (RMSD) in the $\mathrm{m} / \mathrm{z}$ measurement, $\sigma[\mathrm{m} / \mathrm{z}] /[\mathrm{m} / \mathrm{z}]$, is around $0.0054 .^{2}$ The charge RMSD $(\sigma z)$ depends on both the oscillation frequency $(f)$ and the trapping time $\left(t_{\text {trap }}\right)$ and is given approximately by ${ }^{3}$

$$
\sigma z=25.58 \frac{f^{-0.457}}{t_{\text {trap }}{ }^{0.5}}
$$

The oscillation frequency and $\mathrm{m} / \mathrm{z}$ are related through the following equation,

$$
\frac{m}{z}=\frac{C}{f^{2}}
$$

where $\mathrm{C}$ is a constant that depends on the ion energy and trap geometry. For the spike trimer at $475 \mathrm{kDa}$ in Figure 5 of the manuscript the average $\mathrm{m} / \mathrm{z}$ is around $10,000 \mathrm{Da}$, and the average oscillation frequency is around $16,000 \mathrm{~Hz}$. For a 100 ms trapping time, the charge RMSD is 0.97

e, according to Equation 2. This leads to a relative charge RMSD $(\sigma z / z)$ of around 0.0205 . Combining the relative RMSDs in the charge $(\sigma z / z)$ and $\mathrm{m} / \mathrm{z}(\sigma[\mathrm{m} / \mathrm{z}] /[\mathrm{m} / z])$ leads to the relative RMSD in the mass $(\sigma \mathrm{m} / \mathrm{m})$, which is around 0.0212 . Thus, the full width at half maximum (FWHM) of the spike trimer peak at $475 \mathrm{kDa}$ in Figure 5 due to instrumental resolution is $0.0212 \times 2.35 \times 475=23.7 \mathrm{kDa}$, where the factor of 2.35 converts from RMSD to peak FWHM. 


\section{References}

1 N. C. Contino and M. F. Jarrold, Charge Detection Mass Spectrometry for Single Ions With a Limit of Detection of 30 Charges, Int. J. Mass Spectrom. 2013, 345-347, 153-159.

https://doi.org/10.1016/j.ijms.2012.07.010

2 Hogan, J. A.; Jarrold, M. F. Optimized electrostatic linear ion trap for charge detection mass spectrometry. J. Am. Soc. Mass Spectrom. 2018, 29, 2086-2095.

https://doi.org/10.1007/s13361-018-2007-x

3 D. Z. Keifer, T. Motwani, C. M. Teschke, and M. F. Jarrold, Acquiring Structural Information on Virus Particles Via Charge Detection Mass Spectrometry, J. Am. Soc. Mass Spectrom. 2016, 27, 1028-1036. https://doi.org/10.1007/s13361-016-1362-8 